\title{
QED effects on individual atomic orbital energies
}

\author{
Karol Kozioł ${ }^{1, a)}$ and Gustavo A. Aucar ${ }^{1,2, b)}$ \\ ${ }^{1}$ Institute for Modelling and Innovative Technology, IMIT, Corrientes, Argentina \\ ${ }^{2}$ Physics Department, Natural and Exact Science Faculty, Northeastern University of Argentina, \\ Corrientes, Argentina
}

(Received 16 February 2018; accepted 13 March 2018; published online 2 April 2018)

\begin{abstract}
Several issues, concerning QED corrections, that are important in precise atomic calculations are presented. The leading QED corrections, self-energy and vacuum polarization, to the orbital energy for selected atoms with $30 \leq Z \leq 118$ have been calculated. The sum of QED and Breit contributions to the orbital energy is analyzed. It has been found that for $n s$ subshells the Breit and QED contributions are of comparative size, but for $n p$ and $n d$ subshells the Breit contribution takes a major part of the QED+Breit sum. It has also, been found that the Breit to leading QED contributions ratio for $n s$ subshells is almost independent of $Z$. The $Z$-dependence of QED and Breit+QED contributions per subshell is shown. The fitting coefficients may be used to estimate QED effects on inner molecular orbitals. We present results of our calculations for QED contributions to orbital energy of valence $n s$-subshell for group 1 and 11 atoms and discuss about the reliability of these numbers by comparing them with experimental first ionization potential data. Published by AIP Publishing. https://doi.org/10.1063/1.5026193
\end{abstract}

\section{INTRODUCTION}

There is an increasing interest to study the electronic effects on atomic systems that are included in the field called precision tests of bound state QED. ${ }^{1,2}$ Furthermore, accurate calculations of magnetic atomic properties are reaching the stage that some earlier considered vanishingly small effects, as QED need to be included. ${ }^{3-6}$ In addition to the relativistic effects for heavy-atom containing molecules, one should start to consider the effects of the nuclear model and also two-body effects beyond Coulomb interactions and QED effects. ${ }^{7-10}$

The original meaning of the term "Lamb shift" was related to the energy difference between $2 s_{1 / 2}$ and $2 p_{1 / 2}$ levels of the hydrogen atom, which is explained by the QED. ${ }^{11}$ The contemporary use of the term "Lamb shift" is related with the change of energy of any atomic (or ionic or molecular) level due to QED effects. The contribution to the energy levels of QED effects in hydrogen-like atoms have a dependence as $(m c)^{2} \alpha(\alpha Z)^{4} \ln (\alpha Z)$ [or $\alpha(\alpha Z)^{2} \ln (\alpha Z)$ in atomic hartree units] for self-energy (SE) corrections and of $(m c)^{2} \alpha(\alpha Z)^{4}$ [or $\alpha(\alpha Z)^{2}$ in atomic hartree units] for vacuum polarization (VP $)^{2}$ where $m$ is the electron mass, $c$ is the speed of light, $\alpha$ is the fine structure constant, and $Z$ is the nuclear charge. They are leading Lamb shift contributions.

In the past years, some accurate theoretical studies on the value of such contributions were performed though most of them had focus on ionic systems of two-, ${ }^{12}$ three-,${ }^{13}$ or fourelectrons. ${ }^{14}$ On the experimental side, the precise spectroscopy of ions produced in Electron Beam Ion Traps (EBITs) devices has been used in order to test QED in the case of more complex atomic systems, such as Na-like to P-like ${ }^{15,16}$ and $\mathrm{Cu}$-like ${ }^{17}$ high- $Z$ ions. The high-accurate laser spectroscopy of simple one-electron $\mathrm{H}_{2}^{+}$and $\mathrm{HD}^{+}$molecules ${ }^{18,19}$ and two-electron $\mathrm{H}_{2}$, $\mathrm{D}_{2}$, and HD molecules ${ }^{20}$ is used to probe QED corrections. Pyykkö et al. ${ }^{21}$ estimated QED effects on the rovibrational states of water and found that these are significantly larger than current experimental uncertainties, which gives a chance to examine QED effects experimentally.

In the present work, we want to shed some light on several issues concerning QED corrections that are important in precise atomic calculations. We want to answer the following questions: (i) How large is each one of the main QED effects on the orbital energies? (ii) Do they have any functional dependence with $Z$ ? (iii) Are QED effects of the same order as the Breit contributions? (iv) Is the pattern for inner-shell orbitals different than the one for valence orbitals? For this purpose, the leading QED correction, self-energy and vacuum polarization, contributions to the orbital energy for selected atoms with $30 \leq Z \leq 118$ were calculated, by means of MultiConfiguration Dirac-Hartree-Fock (MCDF) codes, and analyzed. We studied QED and Breit contributions to the orbital energy and the $Z$-dependence of QED and Breit+QED contributions per subshell.

\section{THEORETICAL BACKGROUND}

\section{A. MCDF methods}

The methodology of MCDF calculations performed in the present studies is similar to the one published earlier, in several papers (see, e.g., Refs. 22-27). The effective Hamiltonian for an $N$-electron system is expressed by

$$
\hat{H}=\sum_{i=1}^{N} \hat{h}_{D}(i)+\sum_{j>i=1}^{N} V_{i j},
$$

\footnotetext{
b)Electronic mail: gaa@unne.edu.ar
} 
where $\hat{h}_{D}(i)$ is the Dirac one-particle operator for the $i$ th electron and the terms $\hat{V}_{i j}$ account for the effective electronelectron interactions and depend on the photon propagator. The latter are a sum of the Coulomb interaction $\hat{V}_{i j}^{C}$ operator and the transverse Breit $\hat{V}_{i j}^{B}$ operator, ${ }^{28-30}$

$$
\hat{V}_{i j}=\hat{V}_{i j}^{C}+\hat{V}_{i j}^{B},
$$

where the Coulomb interaction operator is $\hat{V}_{i j}^{C}=1 / r_{i j}$ and the frequency-dependent Breit $\hat{V}_{i j}^{B}$ operator $^{31}$ is written, within the Coulomb gauge, as

$$
\hat{V}_{i j}^{B}=-\boldsymbol{\alpha}_{i} \cdot \boldsymbol{\alpha}_{j} \frac{e^{i \omega_{i j} r_{i j}}}{r_{i j}}-\left(\boldsymbol{\alpha}_{i} \cdot \nabla_{i}\right)\left(\boldsymbol{\alpha}_{j} \cdot \boldsymbol{\nabla}_{j}\right) \frac{e^{i \omega_{i j} r_{i j}}-1}{\omega_{i j}^{2} r_{i j}} .
$$

An atomic state function (ASF) with the total angular momentum $J$, its $z$-projection $M$, and parity $p$ is assumed in the form

$$
\Psi_{s}\left(J M^{p}\right)=\sum_{m} c_{m}(s) \Phi\left(\gamma_{m} J M^{p}\right),
$$

where $\Phi\left(\gamma_{m} J M^{p}\right)$ are configuration state functions (CSF), $c_{m}(s)$ are the configuration mixing coefficients for state $s$, and $\gamma_{m}$ represents all information required to uniquely define a certain CSF.
The CSF is a set of Slater determinants of Dirac 4component bispinors,

$$
\Phi\left(\gamma_{m} J M^{p}\right)=\sum_{i} d_{i}\left|\begin{array}{ccc}
\psi_{1}(1) & \cdots & \psi_{1}(N) \\
\vdots & \ddots & \vdots \\
\psi_{N}(1) & \cdots & \psi_{N}(N)
\end{array}\right|,
$$

where the $\psi_{i}$ are the one-electron wavefunctions and the $d_{i}$ coefficients are determined by requiring that the CSF is an eigenstate of $\hat{J}^{2}$ and $\hat{J}_{z}$. The one-electron wavefunction is defined as

$$
\psi=\frac{1}{r}\left(\begin{array}{c}
P_{n, \kappa}(r) \cdot \Omega_{\kappa, j}^{m_{j}}(\theta, \phi) \\
i Q_{n, \kappa}(r) \cdot \Omega_{-\kappa, j}^{m_{j}}(\theta, \phi)
\end{array}\right),
$$

where $\Omega_{\kappa, j}^{m_{j}}(\theta, \phi)$ is an angular 2-component spinor and $P_{n, \kappa}(r)$ and $Q_{n, \kappa}(r)$ are large and small radial parts of the wavefunction, respectively.

\begin{tabular}{|c|c|c|c|c|c|c|c|}
\hline$Z$ & $1 s_{1 / 2}$ & $2 s_{1 / 2}$ & $2 p_{1 / 2}$ & $2 p_{3 / 2}$ & $3 s_{1 / 2}$ & $3 p_{1 / 2}$ & $3 p_{3 / 2}$ \\
\hline 30 & 0.2466 & 0.0261 & -0.0005 & 0.0012 & 0.0038 & $<0.0001$ & 0.0002 \\
\hline 32 & 0.3073 & 0.0332 & -0.0006 & 0.0016 & 0.0051 & $<0.0001$ & 0.0002 \\
\hline 36 & 0.4587 & 0.0515 & -0.0007 & 0.0028 & 0.0085 & $<0.0001$ & 0.0004 \\
\hline 48 & 1.2205 & 0.1501 & $<0.0001$ & 0.0111 & 0.0292 & 0.0003 & 0.0021 \\
\hline 50 & 1.4033 & 0.1750 & 0.0006 & 0.0135 & 0.0347 & 0.0005 & 0.0026 \\
\hline 54 & 1.8272 & 0.2342 & 0.0024 & 0.0196 & 0.0481 & 0.0011 & 0.0040 \\
\hline 62 & 2.9505 & 0.3989 & 0.0112 & 0.0380 & 0.0861 & 0.0036 & 0.0082 \\
\hline 72 & 5.0228 & 0.7260 & 0.0406 & 0.0781 & 0.1645 & 0.0120 & 0.0180 \\
\hline 80 & 7.4080 & 1.1302 & 0.0940 & 0.1296 & 0.2615 & 0.0269 & 0.0311 \\
\hline 82 & 8.1333 & 1.2582 & 0.1141 & 0.1460 & 0.2892 & 0.0325 & 0.0354 \\
\hline 86 & 9.7600 & 1.5524 & 0.1659 & 0.1835 & 0.3525 & 0.0469 & 0.0452 \\
\hline 104 & 21.6117 & 3.8830 & 0.8416 & 0.4887 & 0.9644 & 0.2511 & 0.1346 \\
\hline 112 & 30.5752 & 5.7672 & 1.6611 & 0.6997 & 1.5061 & 0.5091 & 0.2002 \\
\hline 114 & 33.3693 & 6.3730 & 1.9737 & 0.7628 & 1.6936 & 0.6100 & 0.2199 \\
\hline 118 & 39.7825 & 7.7743 & 2.7853 & 0.9013 & 2.1609 & 0.8812 & 0.2644 \\
\hline$Z$ & $3 d_{3 / 2}$ & $3 d_{5 / 2}$ & $4 s_{1 / 2}$ & $4 p_{1 / 2}$ & $4 p_{3 / 2}$ & $4 d_{3 / 2}$ & $4 d_{5 / 2}$ \\
\hline 30 & $<0.0001$ & $<0.0001$ & 0.0002 & & & & \\
\hline 32 & $<0.0001$ & $<0.0001$ & 0.0003 & $<0.0001$ & $<0.0001$ & & \\
\hline 36 & $<0.0001$ & $<0.0001$ & 0.0009 & $<0.0001$ & $<0.0001$ & & \\
\hline 48 & -0.0002 & 0.0002 & 0.0053 & 0.0001 & 0.0003 & $<0.0001$ & $<0.0001$ \\
\hline 50 & -0.0002 & 0.0003 & 0.0066 & 0.0001 & 0.0004 & $<0.0001$ & $<0.0001$ \\
\hline 54 & -0.0003 & 0.0004 & 0.0101 & 0.0002 & 0.0008 & $<0.0001$ & 0.0001 \\
\hline 62 & -0.0006 & 0.0009 & 0.0197 & 0.0009 & 0.0018 & -0.0001 & 0.0001 \\
\hline 72 & -0.0012 & 0.0021 & 0.0394 & 0.0029 & 0.0041 & -0.0002 & 0.0004 \\
\hline 80 & -0.0018 & 0.0037 & 0.0659 & 0.0069 & 0.0075 & -0.0003 & 0.0007 \\
\hline 82 & -0.0020 & 0.0042 & 0.0738 & 0.0084 & 0.0087 & -0.0003 & 0.0009 \\
\hline 86 & -0.0023 & 0.0054 & 0.0921 & 0.0124 & 0.0114 & -0.0003 & 0.0012 \\
\hline 104 & -0.0029 & 0.0157 & 0.2711 & 0.0740 & 0.0392 & $<0.0001$ & 0.0040 \\
\hline 112 & -0.0014 & 0.0231 & 0.4375 & 0.1551 & 0.0613 & 0.0009 & 0.0064 \\
\hline 114 & -0.0007 & 0.0254 & 0.4925 & 0.1858 & 0.0675 & 0.0013 & 0.0070 \\
\hline 118 & 0.0012 & 0.0304 & 0.6369 & 0.2712 & 0.0827 & 0.0023 & 0.0086 \\
\hline
\end{tabular}

\section{B. QED corrections}

The bound-state vacuum polarization contribution is related to the creation and annihilation of virtual

TABLE I. Self-energy contribution to the orbital energy for selected atoms (hartree unit). 
electron-positron pairs in the field of the nucleus. It is a correction to the photon propagator. The first term of order $\alpha(Z \alpha)$ can be calculated as the expectation value of the Uehling potential. The Uehling potential in the case of finite nuclear size and spherical symmetric nuclear charge distribution $\rho(\vec{r})$ can be expressed as ${ }^{32}$

$$
\begin{aligned}
U(\vec{r})= & -\frac{2}{3} \frac{Z \alpha^{2} \hbar^{2}}{m r} \int_{0}^{\infty} d^{3} r^{\prime} r^{\prime} \rho\left(r^{\prime}\right) \\
& \times\left[K_{0}\left(\frac{2 m c}{\hbar}\left|r-r^{\prime}\right|\right)-K_{0}\left(\frac{2 m c}{\hbar}\left|r+r^{\prime}\right|\right)\right],
\end{aligned}
$$

where the function $K_{0}(x)$ is defined as

$$
K_{0}(x)=\int_{1}^{\infty} d t e^{-x t}\left(\frac{1}{t^{3}}+\frac{1}{2 t^{5}}\right) \sqrt{t^{2}-1}
$$

The higher-order terms have been given by Källén and Sabry ${ }^{33}$ for order $\alpha^{2}(Z \alpha)$ and by Wichmann and Kroll ${ }^{34,35}$ for order $\alpha(Z \alpha){ }^{3}$

Self-energy contribution arises from the interaction of the electron with its own radiation field. It is a correction to the electron propagator. For one-electron systems, the most important (one-loop) self-energy term of order $\alpha(Z \alpha)$ has been calculated exactly by Mohr ${ }^{36-38}$ and expressed as

$$
\Delta E_{n \kappa}=\frac{\alpha}{\pi} \frac{(Z \alpha)^{4}}{n^{3}} F_{n \kappa}(Z \alpha) m_{e} c^{2},
$$

where $F_{n \kappa}(Z \alpha)$ is a slowly varying function of $Z \alpha$.

The self-energy screening is taken into account by MCDFGME code in the Welton picture. ${ }^{27,39,40}$ In this approach, the self-energy correction for $s$-type Dirac-Fock orbitals is scaled from exact hydrogenic results from the following relation:

$$
\left(\Delta E_{n s}\right)_{D F}=\frac{\left\langle n s\left|\nabla^{2} V_{n u c l}(r)\right| n s\right\rangle_{D F}}{\left\langle n s\left|\nabla^{2} V_{n u c l}(r)\right| n s\right\rangle_{H y d}}\left(\Delta E_{n s}\right)_{H y d},
$$

where $V_{n u c l}(r)$ is a nuclear potential. For $l \geq 1$ subshells, the following expression is used: ${ }^{40}$

\begin{tabular}{|c|c|c|c|c|c|c|c|}
\hline$Z$ & $1 s_{1 / 2}$ & $2 s_{1 / 2}$ & $2 p_{1 / 2}$ & $2 p_{3 / 2}$ & $3 s_{1 / 2}$ & $3 p_{1 / 2}$ & $3 p_{3 / 2}$ \\
\hline 30 & -0.0231 & -0.0023 & $<0.0001$ & $<0.0001$ & -0.0003 & $<0.0001$ & $<0.0001$ \\
\hline 32 & -0.0299 & -0.0030 & $<0.0001$ & $<0.0001$ & -0.0004 & $<0.0001$ & $<0.0001$ \\
\hline 36 & -0.0483 & -0.0050 & -0.0001 & $<0.0001$ & -0.0008 & $<0.0001$ & $<0.0001$ \\
\hline 48 & -0.1589 & -0.0180 & -0.0006 & -0.0001 & -0.0034 & -0.0001 & $<0.0001$ \\
\hline 50 & -0.1889 & -0.0217 & -0.0008 & -0.0001 & -0.0042 & -0.0002 & $<0.0001$ \\
\hline 54 & -0.2627 & -0.0311 & -0.0013 & -0.0001 & -0.0062 & -0.0003 & $<0.0001$ \\
\hline 62 & -0.4824 & -0.0603 & -0.0036 & -0.0003 & -0.0128 & -0.0008 & -0.0001 \\
\hline 72 & -0.9615 & -0.1294 & -0.0110 & -0.0008 & -0.0287 & -0.0027 & -0.0002 \\
\hline 80 & -1.6100 & -0.2306 & -0.0257 & -0.0016 & -0.0525 & -0.0066 & -0.0004 \\
\hline 82 & -1.8251 & -0.2657 & -0.0316 & -0.0018 & -0.0609 & -0.0081 & -0.0005 \\
\hline 86 & -2.3378 & -0.3520 & -0.0474 & -0.0024 & -0.0815 & -0.0123 & -0.0007 \\
\hline 104 & -6.9871 & -1.2483 & -0.2898 & -0.0078 & -0.3001 & -0.0783 & -0.0023 \\
\hline 112 & -11.4416 & -2.2283 & -0.6632 & -0.0122 & -0.5410 & -0.1801 & -0.0038 \\
\hline 114 & -12.9681 & -2.5826 & -0.8203 & -0.0137 & -0.6281 & -0.2228 & -0.0043 \\
\hline 118 & -16.7082 & -3.4810 & -1.2661 & -0.0169 & -0.8487 & -0.3430 & -0.0054 \\
\hline$Z$ & $3 d_{3 / 2}$ & $3 d_{5 / 2}$ & $4 s_{1 / 2}$ & $4 p_{1 / 2}$ & $4 p_{3 / 2}$ & $4 d_{3 / 2}$ & $4 d_{5 / 2}$ \\
\hline 30 & $<0.0001$ & $<0.0001$ & $<0.0001$ & & & & \\
\hline 32 & $<0.0001$ & $<0.0001$ & $<0.0001$ & $<0.0001$ & $<0.0001$ & & \\
\hline 36 & $<0.0001$ & $<0.0001$ & -0.0001 & $<0.0001$ & $<0.0001$ & & \\
\hline 48 & $<0.0001$ & $<0.0001$ & -0.0006 & $<0.0001$ & $<0.0001$ & $<0.0001$ & $<0.0001$ \\
\hline 50 & $<0.0001$ & $<0.0001$ & -0.0008 & $<0.0001$ & $<0.0001$ & $<0.0001$ & $<0.0001$ \\
\hline 54 & $<0.0001$ & $<0.0001$ & -0.0013 & -0.0001 & $<0.0001$ & $<0.0001$ & $<0.0001$ \\
\hline 62 & $<0.0001$ & $<0.0001$ & -0.0029 & -0.0002 & $<0.0001$ & $<0.0001$ & $<0.0001$ \\
\hline 72 & $<0.0001$ & $<0.0001$ & -0.0068 & -0.0006 & $<0.0001$ & $<0.0001$ & $<0.0001$ \\
\hline 80 & $<0.0001$ & $<0.0001$ & -0.0132 & -0.0016 & -0.0001 & $<0.0001$ & $<0.0001$ \\
\hline 82 & $<0.0001$ & $<0.0001$ & -0.0154 & -0.0020 & -0.0001 & $<0.0001$ & $<0.0001$ \\
\hline 86 & $<0.0001$ & $<0.0001$ & -0.0212 & -0.0032 & -0.0002 & $<0.0001$ & $<0.0001$ \\
\hline 104 & -0.0001 & $<0.0001$ & -0.0845 & -0.0221 & -0.0007 & $<0.0001$ & $<0.0001$ \\
\hline 112 & -0.0001 & $<0.0001$ & -0.1560 & -0.0523 & -0.0011 & $<0.0001$ & $<0.0001$ \\
\hline 114 & -0.0001 & $<0.0001$ & -0.1821 & -0.0650 & -0.0013 & -0.0001 & $<0.0001$ \\
\hline 118 & -0.0002 & $<0.0001$ & -0.2482 & -0.1010 & -0.0017 & -0.0001 & $<0.0001$ \\
\hline
\end{tabular}

$$
\left(\Delta E_{n \kappa}\right)_{D F}=\frac{\langle n \kappa|\beta \boldsymbol{\alpha} \boldsymbol{E}| n \kappa\rangle_{D F}}{\langle n \kappa|\beta \boldsymbol{\alpha} \boldsymbol{E}| n \kappa\rangle_{H y d}}\left(\Delta E_{n \kappa}\right)_{H y d},
$$

TABLE II. Vacuum polarization (according to Uehling potential) contribution to the orbital energy for selected atoms (hartree unit). 
where $\boldsymbol{E}$ is the nuclear electric field.

Another way to calculate SE contributions, implemented in older versions of Grasp code, is using the scaling law obtained by comparing the mean value of the radial coordinate of Dirac-Fock radial wavefunction $\langle r\rangle_{D F}$ to the hydrogenic one $\left\langle r\left(Z_{\text {eff }}\right)\right\rangle_{H y d}$. Both approaches give similar results for SE contributions for the inner shell, but some papers (e.g., Ref. 41) indicate that the Welton picture gives more reliable SE values for the outer shell of high- $Z$ atoms. The improved version of GRASP code, the GRASP2K package, approximates the screening coefficient by taking the ratio of the Dirac-Fock wavefunction density in a small region around the nucleus $\left(r \leq r^{\prime}\right.$, $r^{\prime}=0.0219 a_{0}, a_{0}$-Bohr's radius) to the equivalent density for a hydrogenic orbital, i.e., ${ }^{42}$

$$
\left(\Delta E_{n \kappa}\right)_{D F}=\frac{\left\langle n \kappa_{r \leq r^{\prime}} \mid n \kappa_{r \leq r^{\prime}}\right\rangle_{D F}}{\left\langle n \kappa_{r \leq r^{\prime}} \mid n \kappa_{r \leq r^{\prime}}\right\rangle_{H y d}}\left(\Delta E_{n \kappa}\right)_{H y d} .
$$

This method gives results closer to those obtained by using the Welton picture. ${ }^{42}$ Lowe et al. ${ }^{42}$ created extension of the Grasp2K package that implements the Welton picture approach to estimate SE screening into GRASP2K suite.
In the last few years, some modern approaches for the estimation of hydrogenic SE data to many-electron atoms have been presented, such as the model Lamb shift operator (MLSO) $)^{43-46}$ and the spectral representation (projection operator) of the Lamb shift. ${ }^{47}$

\section{Computational details}

The leading QED contributions, listed below, to orbital energies have been calculated in the present work by using McDFGME code. For the VP contributions, the Uehling potential [of order $\alpha(Z \alpha)$ ], the Källén and Sabry potential [of order $\alpha^{2}(Z \alpha)$ ], and the Wichmann and Kroll potential [of order $\alpha(Z \alpha)^{3}$ ] have been used in first-order perturbation with DiracFock wavefunctions. ${ }^{48}$ Screened SE contribution of order $\alpha(Z \alpha)$ has been obtained by using the Welton picture. For $Z$ $=104,112,114$, and 118, we used earlier calculated SE results from Grasp2K code assisted by Lowe et al.'s ${ }^{42}$ extension.

Present results for Breit interaction contribution were obtained by performing calculations in the self-consistent approach with McDFGME ${ }^{49-51}$ code. See our previous paper for details. $^{52}$

TABLE III. The leading QED contribution to the orbital energy for selected atoms (hartree unit).

\begin{tabular}{|c|c|c|c|c|c|c|c|}
\hline$Z$ & $1 s_{1 / 2}$ & $2 s_{1 / 2}$ & $2 p_{1 / 2}$ & $2 p_{3 / 2}$ & $3 s_{1 / 2}$ & $3 p_{1 / 2}$ & $3 p_{3 / 2}$ \\
\hline 30 & 0.2236 & 0.0239 & -0.0005 & 0.0012 & 0.0035 & $<0.0001$ & 0.0002 \\
\hline 32 & 0.2774 & 0.0302 & -0.0006 & 0.0016 & 0.0046 & -0.0001 & 0.0002 \\
\hline 36 & 0.4106 & 0.0465 & -0.0008 & 0.0028 & 0.0077 & -0.0001 & 0.0004 \\
\hline 48 & 1.0632 & 0.1323 & -0.0006 & 0.0111 & 0.0258 & 0.0002 & 0.0021 \\
\hline 50 & 1.2166 & 0.1535 & -0.0002 & 0.0135 & 0.0305 & 0.0003 & 0.0026 \\
\hline 54 & 1.5683 & 0.2035 & 0.0012 & 0.0194 & 0.0419 & 0.0008 & 0.0039 \\
\hline 62 & 2.4779 & 0.3398 & 0.0077 & 0.0377 & 0.0735 & 0.0028 & 0.0082 \\
\hline 72 & 4.0877 & 0.6000 & 0.0300 & 0.0773 & 0.1366 & 0.0094 & 0.0178 \\
\hline 80 & 5.8515 & 0.9069 & 0.0695 & 0.1282 & 0.2106 & 0.0206 & 0.0307 \\
\hline 82 & 6.3714 & 1.0012 & 0.0841 & 0.1444 & 0.2303 & 0.0248 & 0.0349 \\
\hline 86 & 7.5096 & 1.2128 & 0.1209 & 0.1814 & 0.2738 & 0.0352 & 0.0446 \\
\hline 104 & 14.9647 & 2.6909 & 0.5704 & 0.4824 & 0.6776 & 0.1777 & 0.1327 \\
\hline 112 & 19.7381 & 3.6472 & 1.0424 & 0.6900 & 0.9910 & 0.3407 & 0.1971 \\
\hline 114 & 21.0985 & 3.9180 & 1.2090 & 0.7519 & 1.0961 & 0.4019 & 0.2165 \\
\hline 118 & 24.0022 & 4.4707 & 1.6064 & 0.8880 & 1.3549 & 0.5610 & 0.2602 \\
\hline$Z$ & $3 d_{3 / 2}$ & $3 d_{5 / 2}$ & $4 s_{1 / 2}$ & $4 p_{1 / 2}$ & $4 p_{3 / 2}$ & $4 d_{3 / 2}$ & $4 d_{5 / 2}$ \\
\hline 30 & $<0.0001$ & $<0.0001$ & 0.0001 & & & & \\
\hline 32 & $<0.0001$ & $<0.0001$ & 0.0003 & $<0.0001$ & $<0.0001$ & & \\
\hline 36 & $<0.0001$ & $<0.0001$ & 0.0008 & $<0.0001$ & $<0.0001$ & & \\
\hline 48 & -0.0002 & 0.0002 & 0.0047 & $<0.0001$ & 0.0003 & $<0.0001$ & $<0.0001$ \\
\hline 50 & -0.0002 & 0.0003 & 0.0058 & 0.0001 & 0.0004 & $<0.0001$ & $<0.0001$ \\
\hline 54 & -0.0003 & 0.0004 & 0.0088 & 0.0002 & 0.0008 & $<0.0001$ & 0.0001 \\
\hline 62 & -0.0006 & 0.0009 & 0.0168 & 0.0007 & 0.0017 & -0.0001 & 0.0001 \\
\hline 72 & -0.0012 & 0.0021 & 0.0327 & 0.0023 & 0.0040 & -0.0002 & 0.0004 \\
\hline 80 & -0.0018 & 0.0037 & 0.0531 & 0.0053 & 0.0074 & -0.0003 & 0.0007 \\
\hline 82 & -0.0020 & 0.0042 & 0.0588 & 0.0064 & 0.0085 & -0.0003 & 0.0009 \\
\hline 86 & -0.0023 & 0.0054 & 0.0716 & 0.0093 & 0.0113 & -0.0003 & 0.0012 \\
\hline 104 & -0.0029 & 0.0157 & 0.1903 & 0.0532 & 0.0387 & -0.0001 & 0.0040 \\
\hline 112 & -0.0015 & 0.0231 & 0.2889 & 0.1061 & 0.0604 & 0.0009 & 0.0064 \\
\hline 114 & -0.0008 & 0.0254 & 0.3192 & 0.1250 & 0.0665 & 0.0012 & 0.0070 \\
\hline 118 & 0.0011 & 0.0303 & 0.4011 & 0.1769 & 0.0814 & 0.0022 & 0.0086 \\
\hline
\end{tabular}




\section{RESULTS}

\section{A. Z-dependence of QED correction}

In Tables I and II, the SE and VP contributions to the orbital energies for selected atoms with $30 \leq \mathrm{Z} \leq 118$ have been collected, respectively. These VP data are related to the Uehling potential only. From both tables, it is interesting to see that the VP contribution compared with the SE one grows up as $Z$ grows for $n s_{1 / 2}$ and $n p_{1 / 2}$ orbitals. For $Z=30, \varepsilon_{n s}^{V P} \simeq 10 \%$ of $\varepsilon_{n s}^{\mathrm{SE}}$, but for $Z=80$ the percentage grows to $\sim 20 \%$ and for $Z=118$ it is of $\sim 40 \%$. A similar behavior is observed for $n p_{1 / /}$ subshells. For all other subshells, the VP contribution is very small compared with SE ones.

In Table III, the leading QED contributions have been collected-see Sec. II C for details. It is worth to mention that, for $Z=86, \varepsilon_{n p_{1 / 2}}^{Q E D}<\varepsilon_{n p_{3 / 2}}^{Q E D}$, but for $Z=104$ this behavior is changed, i.e., $\varepsilon_{n p_{1 / 2}}^{Q E D}>\varepsilon_{n p_{3 / 2}}^{Q E D}$. This behavior is seen in Figs. 1(b)-1(d) and it is similar to that provided by Johnson and Soff ${ }^{53}$ and by Yerokhin and Shabaev ${ }^{54}$ for H-like

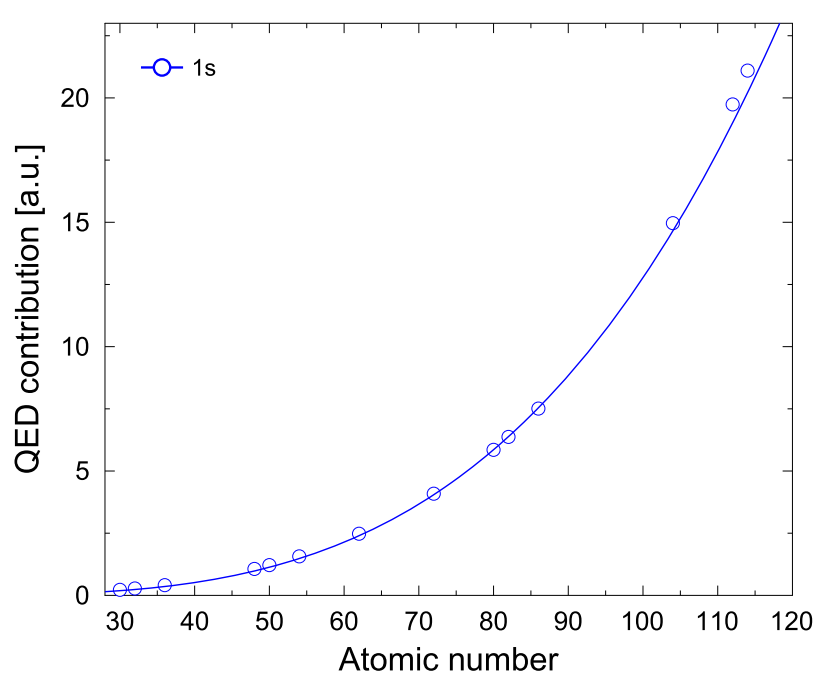

(a)

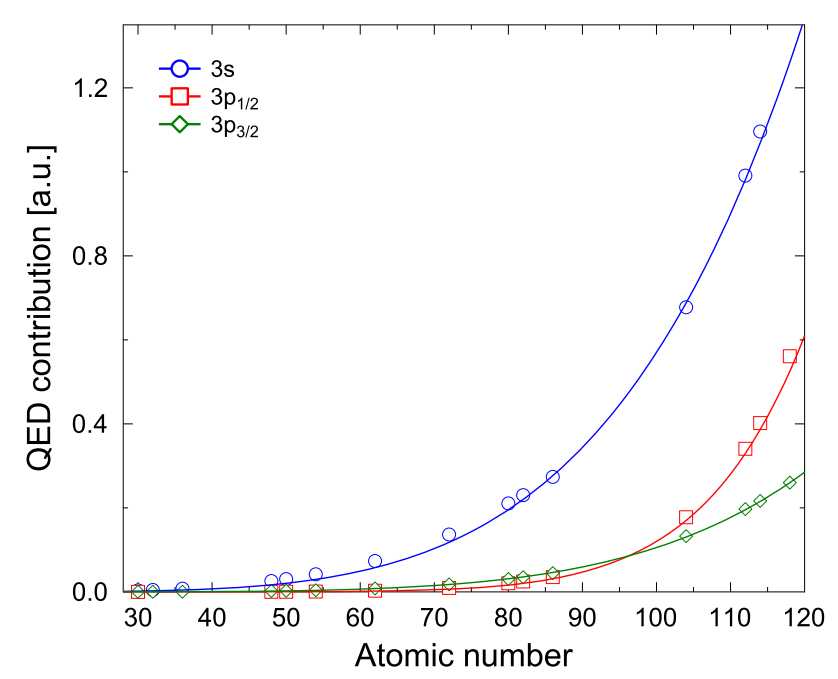

(c) atomic systems, where the Lamb shift contribution to the energy difference between $2 p_{3 / 2}$ and $2 p_{1 / 2}$ orbitals changes a sign between $Z=87$ and 88 . The increase of $\varepsilon_{n p_{1 / 2}}^{Q E D}$ over $\varepsilon_{n p_{3 / 2}}^{Q E D}$ is related to the increase of the $s$-character of the small component of the $n p_{1 / 2}$ orbital. The very rough estimate of small-to-large component ratio is $\sim Z \alpha$, so for high $Z$ the $n p_{1 / 2}$ orbitals behave increasingly like $n s_{1 / 2}$ orbitals. It is worth to mention that in the original Bethe paper ${ }^{55}$ the Lamb shift is linked to the electron density at the nucleus and this value is much larger for $s$-type orbitals than for $p$-type orbitals.

The $Z$-dependence of the $S E$ terms has been examined by fitting SE contributions to the orbital $n s(n=1-4)$ and $n^{\prime} p_{1 / 2}$ and $n^{\prime} p_{3 / 2}\left(n^{\prime}=2-4\right)$ energies by the

$$
\varepsilon_{n l}^{S E}(Z)=a \times Z^{b}
$$

function. Coefficients $a$ and $b$ have been collected in Table IV (also $R^{2}$ fitting parameter has been presented). The fitting

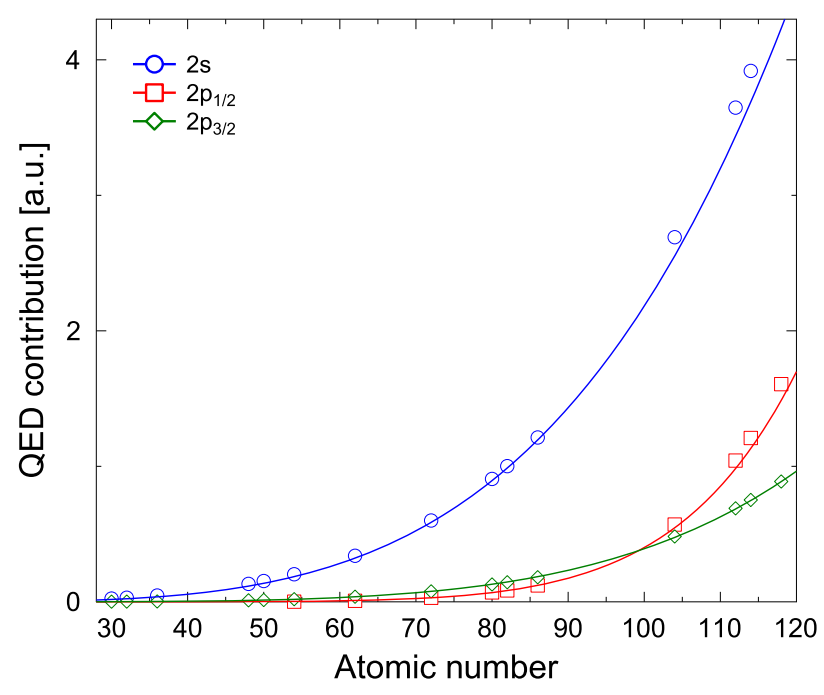

(b)

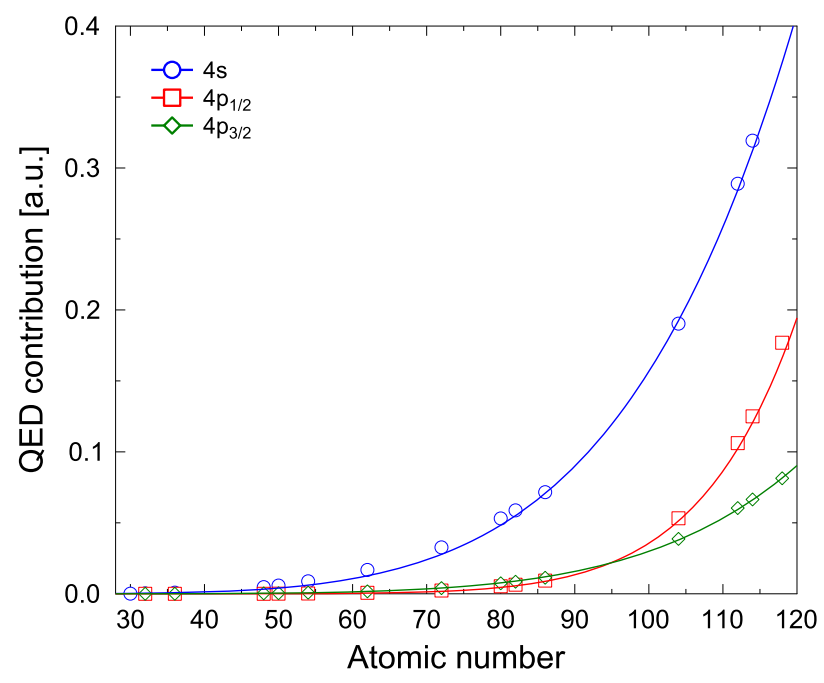

(d)

FIG. 1. The leading QED contributions to the Dirac-Coulomb orbital energy for $n l$ subshells (points) and fit lines to them. 
TABLE IV. Fitting coefficients for $Z$-dependence of SE correction to the orbital energy (see text for details).

\begin{tabular}{lccc}
\hline \hline Subshell & $a$ & $b$ & $R^{2}$ \\
\hline $1 s$ & $4.165 \times 10^{-8}$ & 4.330 & 0.99878 \\
$2 s$ & $2.774 \times 10^{-10}$ & 5.038 & 0.99899 \\
$3 s$ & $3.081 \times 10^{-12}$ & 5.711 & 0.99773 \\
$4 s$ & $8.411 \times 10^{-14}$ & 6.211 & 0.99831 \\
\hline $2 p_{1 / 2}$ & $6.986 \times 10^{-20}$ & 9.458 & 0.99966 \\
$3 p_{1 / 2}$ & $1.717 \times 10^{-21}$ & 9.994 & 0.99951 \\
$4 p_{1 / 2}$ & $9.104 \times 10^{-23}$ & 10.363 & 0.99970 \\
\hline $2 p_{3 / 2}$ & $4.648 \times 10^{-11}$ & 4.966 & 0.99994 \\
$3 p_{3 / 2}$ & $1.203 \times 10^{-12}$ & 5.475 & 0.99993 \\
$4 p_{3 / 2}$ & $2.039 \times 10^{-14}$ & 6.087 & 0.99983 \\
\hline \hline
\end{tabular}

have been performed by means of the Nelder-Mead simplex algorithm implemented in the SciDAVis program. ${ }^{56}$

The exponential dependence of Eq. (13) is larger than 4 in all cases and increases with the principal quantum number, being the largest for $p_{1 / 2}$ subshells. The $b$ coefficient is in the range of 4.330-6.211 for $n s$ subshells, of 4.966-6.087 for $n p_{3 / 2}$ subshells, and of $9.458-10.363$ for $n p_{1 / 2}$ subshells. It can be seen that the $a$ coefficient of $n p_{1 / 2}$ is much smaller than the other two. On the other hand, the more to the valence region one goes, the larger its exponential dependence is. When VP effects are added to SE, the exponential $Z$-dependence of QED effects is smaller, especially for $n p_{1 / 2}$ orbitals, though its behavior is similar to that of SE-see Table V. For total QED effect, the $b$ coefficient is in the range of 3.607-5.398 for $n s$ subshells, of 4.955-6.079 for $n p_{3 / 2}$ subshells, and of 8.191-9.544 for $n p_{1 / 2}$ subshells.

In Fig. 2, the QED energy contributions to Dirac-Coulomb orbital energy ratios $\left(R^{Q E D / D C}\right)$ for selected subshells are presented. It is found that the absolute value of ratio $R^{Q E D / D C}$ increases when $Z$ increases. It is worth to note that for the internal $s$ subshells the $R^{Q E D / D C}$ values for given $Z$ are similar to each other. For example, for $1 s$ subshell, the $R^{Q E D / D C}$ values are $-2.79 \times 10^{-3},-2.84 \times 10^{-3}$, and $-2.93 \times 10^{-3}$ for $Z=112,114$, and 118, respectively. For $2 s$ subshells, they are $-2.52 \times 10^{-3},-2.56 \times 10^{-3}$, and $-2.60 \times 10^{-3}$ and for $3 s$

TABLE V. Fitting coefficients for Z-dependence of QED correction to the orbital energy (see text for details).

\begin{tabular}{lccc}
\hline \hline Subshell & $a$ & $b$ & $R^{2}$ \\
\hline $1 s$ & $8.020 \times 10^{-7}$ & 3.607 & 0.99984 \\
$2 s$ & $1.568 \times 10^{-8}$ & 4.082 & 0.99990 \\
$3 s$ & $8.182 \times 10^{-11}$ & 4.926 & 0.99831 \\
$4 s$ & $2.562 \times 10^{-12}$ & 5.398 & 0.99869 \\
\hline $2 p_{1 / 2}$ & $1.715 \times 10^{-17}$ & 8.191 & 0.99999 \\
$3 p_{1 / 2}$ & $7.619 \times 10^{-20}$ & 9.104 & 0.99970 \\
$4 p_{1 / 2}$ & $2.961 \times 10^{-21}$ & 9.544 & 0.99984 \\
\hline $2 p_{3 / 2}$ & $4.819 \times 10^{-11}$ & 4.955 & 0.99994 \\
$3 p_{3 / 2}$ & $1.237 \times 10^{-12}$ & 5.466 & 0.99992 \\
$4 p_{3 / 2}$ & $2.085 \times 10^{-14}$ & 6.079 & 0.99982 \\
\hline \hline
\end{tabular}

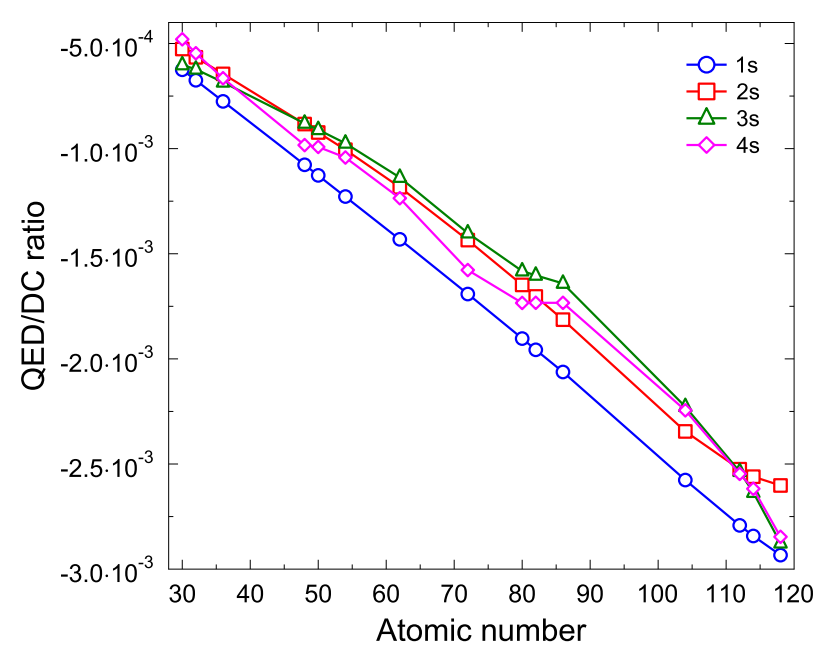

(a)

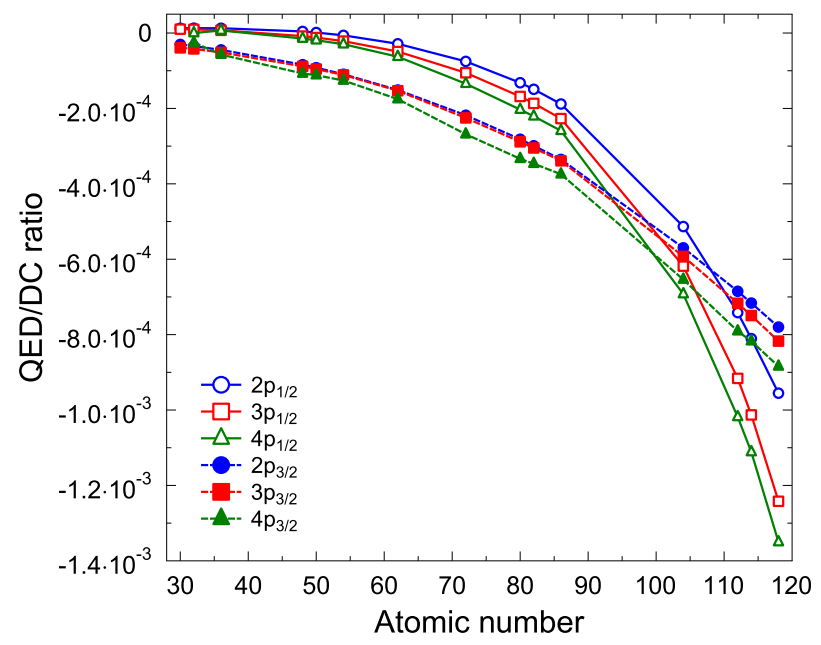

(b)

FIG. 2. The leading QED energy contributions to the Dirac-Coulomb orbital energy ratio for $s$ and $p$ subshells.

subshells they are $-2.54 \times 10^{-3},-2.64 \times 10^{-3}$, and -2.88 $\times 10^{-3}$ for $Z=112,114$, and 118 , respectively. These values are in agreement (relating to Koopman's theorem) with QED contributions to the $1 s$ and $2 s$ ionization potentials (IPs) of the $Z=112,114$, and 118 neutral atoms. ${ }^{57}$

\section{B. Sum of QED and Breit contribution}

In Table VI, the sum of the leading QED plus Breit contributions to the Dirac-Coulomb orbital energy for selected atoms with $30 \leq Z \leq 118$ has been collected. We show in Table VII the $Z$-dependence of QED+Breit effects on neutral atoms. Coefficients $a$ and $b$ are defined in a similar way as in Eq. (13).

The leading QED+Breit contributions to orbital energy for $n l$ subshells (points) and fit lines to them are presented in Fig. 3. It is worth noticing that the QED+Breit sums for $n s$ and for $n p_{1 / 2}$ are of similar size for given $n$, and about twice of the corresponding QED+Breit sum for $n p_{3 / 2}$. Leading QED contribution to the orbital energy for $n s$ subshell is larger than for $n p_{1 / 2}$ subshell, and for Breit contribution the trend 
TABLE VI. Sum of leading QED plus Breit contribution to the orbital energy for selected atoms (hartree unit).

\begin{tabular}{|c|c|c|c|c|c|c|c|}
\hline$Z$ & $1 s_{1 / 2}$ & $2 s_{1 / 2}$ & $2 p_{1 / 2}$ & $2 p_{3 / 2}$ & $3 s_{1 / 2}$ & $3 p_{1 / 2}$ & $3 p_{3 / 2}$ \\
\hline 30 & 0.6600 & 0.0551 & 0.0547 & 0.0370 & 0.0068 & 0.0059 & 0.0033 \\
\hline 32 & 0.8154 & 0.0696 & 0.0692 & 0.0472 & 0.0085 & 0.0074 & 0.0041 \\
\hline 36 & 1.1999 & 0.1074 & 0.1066 & 0.0739 & 0.0139 & 0.0122 & 0.0070 \\
\hline 48 & 3.0802 & 0.3133 & 0.3111 & 0.2220 & 0.0504 & 0.0460 & 0.0295 \\
\hline 50 & 3.5212 & 0.3640 & 0.3614 & 0.2582 & 0.0596 & 0.0545 & 0.0350 \\
\hline 54 & 4.5344 & 0.4841 & 0.4806 & 0.3434 & 0.0824 & 0.0755 & 0.0490 \\
\hline 62 & 7.1538 & 0.8132 & 0.8078 & 0.5736 & 0.1518 & 0.1415 & 0.0937 \\
\hline 72 & 11.7701 & 1.4271 & 1.4213 & 0.9840 & 0.2848 & 0.2666 & 0.1756 \\
\hline 80 & 16.8149 & 2.1364 & 2.1367 & 1.4331 & 0.4382 & 0.4140 & 0.2654 \\
\hline 82 & 18.3005 & 2.3519 & 2.3554 & 1.5645 & 0.4820 & 0.4595 & 0.2920 \\
\hline 86 & 21.5686 & 2.8376 & 2.8521 & 1.8547 & 0.5822 & 0.5651 & 0.3529 \\
\hline 104 & 42.2964 & 6.1613 & 6.5031 & 3.6912 & 1.4028 & 1.4041 & 0.7769 \\
\hline 112 & 55.8102 & 8.4523 & 9.3270 & 4.8142 & 2.0300 & 2.0867 & 1.0457 \\
\hline 114 & 59.7141 & 9.1283 & 10.2200 & 5.1291 & 2.2314 & 2.3086 & 1.1214 \\
\hline 118 & 68.2048 & 10.6040 & 12.2945 & 5.8071 & 2.7143 & 2.8429 & 1.2890 \\
\hline$Z$ & $3 d_{3 / 2}$ & $3 d_{5 / 2}$ & $4 s_{1 / 2}$ & $4 p_{1 / 2}$ & $4 p_{3 / 2}$ & $4 d_{3 / 2}$ & $4 d_{5 / 2}$ \\
\hline 30 & 0.0002 & -0.0008 & 0.0003 & & & & \\
\hline 32 & 0.0001 & -0.0012 & 0.0004 & 0.0001 & 0.0003 & & \\
\hline 36 & 0.0007 & -0.0016 & 0.0010 & 0.0006 & 0.0001 & & \\
\hline 48 & 0.0121 & 0.0044 & 0.0081 & 0.0064 & 0.0035 & 0.0001 & -0.0009 \\
\hline 50 & 0.0150 & 0.0059 & 0.0097 & 0.0077 & 0.0041 & -0.0001 & -0.0014 \\
\hline 54 & 0.0228 & 0.0106 & 0.0144 & 0.0117 & 0.0063 & 0.0002 & -0.0019 \\
\hline 62 & 0.0529 & 0.0306 & 0.0319 & 0.0284 & 0.0161 & 0.0077 & $<0.0001$ \\
\hline 72 & 0.1078 & 0.0705 & 0.0629 & 0.0551 & 0.0329 & 0.0140 & 0.0061 \\
\hline 80 & 0.1690 & 0.1136 & 0.0998 & 0.0887 & 0.0510 & 0.0227 & 0.0103 \\
\hline 82 & 0.1871 & 0.1263 & 0.1104 & 0.0992 & 0.0562 & 0.0250 & 0.0112 \\
\hline 86 & 0.2291 & 0.1563 & 0.1362 & 0.1253 & 0.0697 & 0.0321 & 0.0150 \\
\hline 104 & 0.5244 & 0.3727 & 0.3704 & 0.3610 & 0.1821 & 0.0981 & 0.0591 \\
\hline 112 & 0.7118 & 0.5072 & 0.5566 & 0.5596 & 0.2540 & 0.1394 & 0.0855 \\
\hline 114 & 0.7641 & 0.5440 & 0.6147 & 0.6238 & 0.2731 & 0.1503 & 0.0918 \\
\hline 118 & 0.8801 & 0.6262 & 0.7621 & 0.7838 & 0.3194 & 0.1773 & 0.1091 \\
\hline
\end{tabular}

is reversed-Breit contribution to the $n p_{1 / 2}$ orbital energy is larger than to the $n s$ one. Both differences complement one another and, as a result, the QED+Breit contribution sums for $n s$ and for $n p_{1 / 2}$ are almost equal.

It is also worth to mention that for $n s$ subshells the Breit and QED contributions are of comparative size, but for $n p$

TABLE VII. Fitting coefficients for the $Z$-dependence of the sum of leading QED plus Breit corrections to the orbital energy (see text for details).

\begin{tabular}{lccc}
\hline \hline Subshell & $a$ & $b$ & $R^{2}$ \\
\hline $1 s$ & $2.391 \times 10^{-6}$ & 3.600 & 0.99970 \\
$2 s$ & $3.436 \times 10^{-8}$ & 4.100 & 0.99966 \\
$3 s$ & $2.561 \times 10^{-10}$ & 4.838 & 0.99900 \\
$4 s$ & $4.208 \times 10^{-12}$ & 5.434 & 0.99926 \\
\hline $2 p_{1 / 2}$ & $5.741 \times 10^{-9}$ & 4.500 & 0.99871 \\
$3 p_{1 / 2}$ & $7.468 \times 10^{-11}$ & 5.104 & 0.99871 \\
$4 p_{1 / 2}$ & $6.715 \times 10^{-13}$ & 5.823 & 0.99918 \\
\hline $2 p_{3 / 2}$ & $1.968 \times 10^{-7}$ & 3.606 & 0.99999 \\
$3 p_{3 / 2}$ & $4.558 \times 10^{-9}$ & 4.080 & 0.99996 \\
$4 p_{3 / 2}$ & $5.645 \times 10^{-11}$ & 4.709 & 0.99970 \\
\hline
\end{tabular}

and $n d$ subshells the Breit contribution takes major part of the QED+Breit sum-see Fig. 4. For $1 s$ and $2 s$ subshells, the contributions of Breit and leading QED are almost constant to their addition. For $1 s$, the leading QED contribution is $\approx 30 \%$ and for $2 s$ such contribution is $\approx 42 \%$ of the QED+Breit sum. It is interesting to see that the SE contributes with a higher and positive percentage for higher $Z$, and VP contribution has an opposite behavior.

It is worth to mention that for muonic atoms the dominant QED contribution to Lamb shift is VP. ${ }^{58}$

\section{QED and Breit contributions for ns subshells}

It is very interesting to observe the behavior of Breit to leading QED contributions ratio $\left(R^{B / Q E D}\right)$ as $Z$ increases for $n s$ subshells. In Fig. 5, one can see that for inner shells the $R^{B / Q E D}$ ratio is almost independent of $Z$ : for $1 s$ subshells, it is about 1.9-2.0; for $2 s$ subshells, it is about 1.3-1.5; and for $3 s$ subshells, it is about 1.0-1.1. Pyykkö et al. ${ }^{59}$ drew a conclusion that for valence $n s$ shells of group 1 and group 11 metal atoms the Lamb shift is roughly half of the Breit contribution and this ratio is almost independent of $Z$. Our results for valence $n s$ shells are much different-the QED 


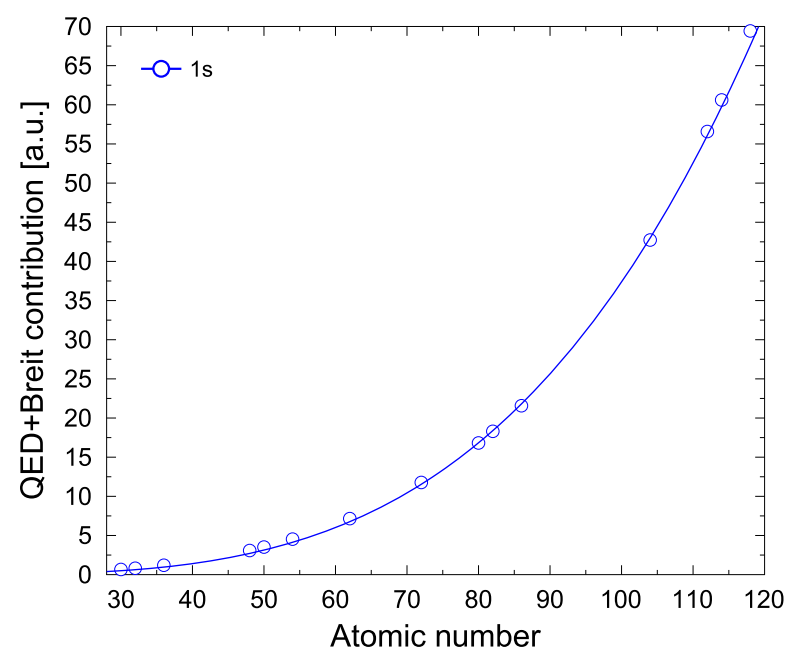

(a)

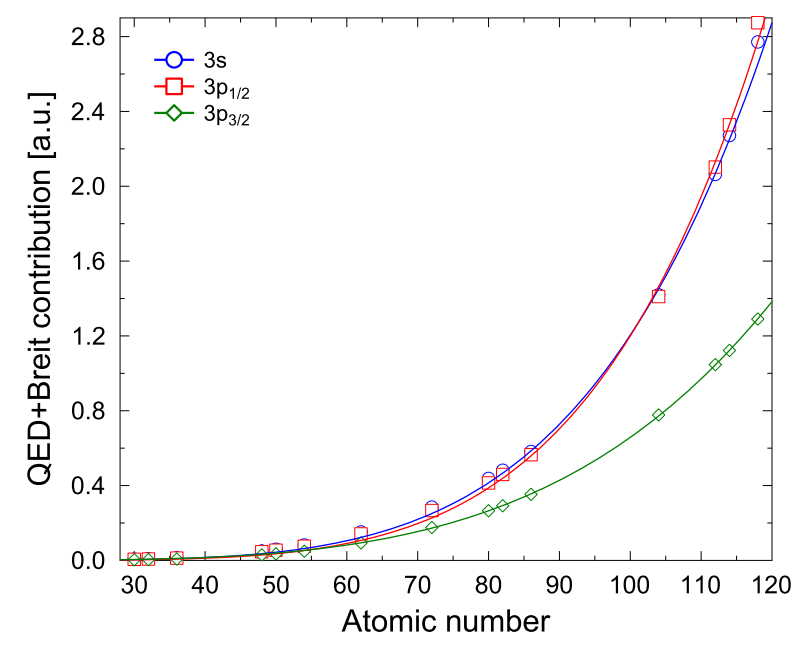

(c)

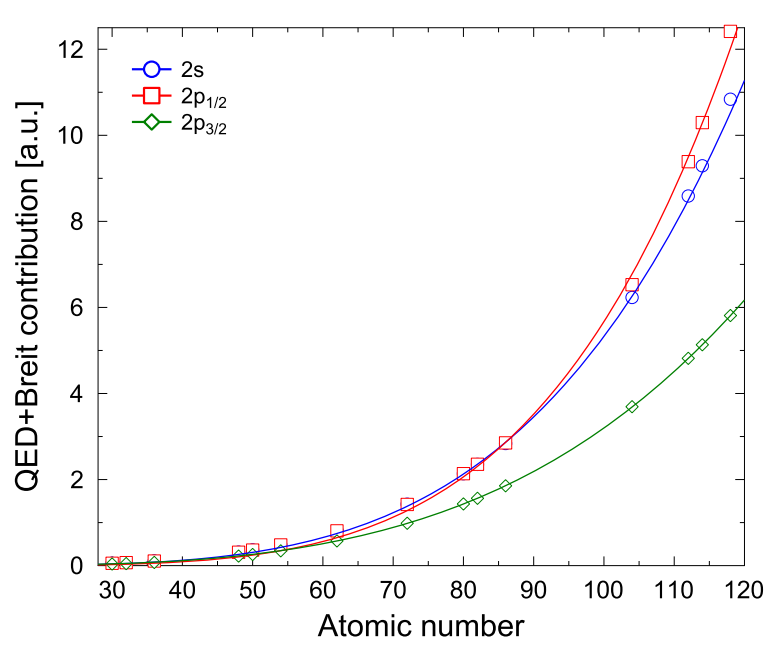

(b)

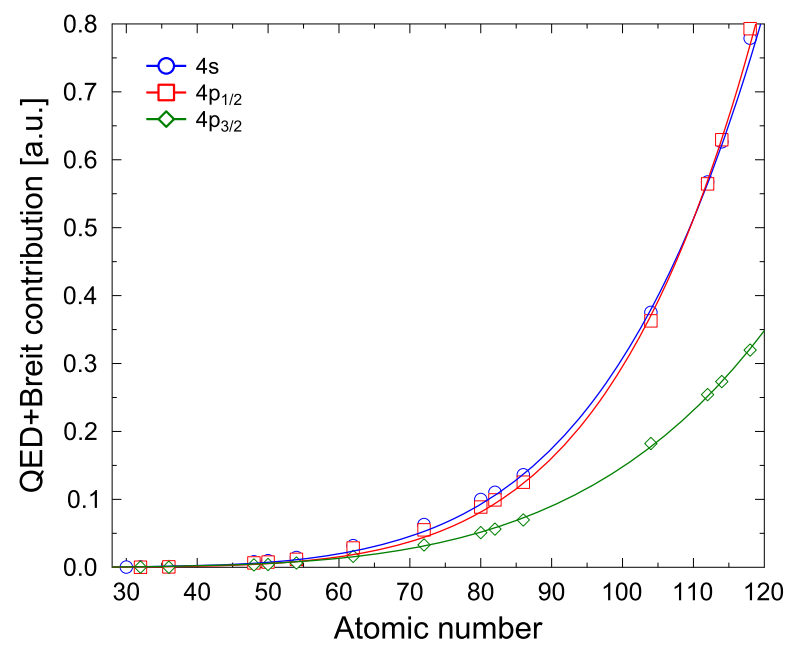

(d)

FIG. 3. Sum of leading QED plus Breit contributions to the Dirac-Coulomb orbital energy for $n l$ subshells (points) and fit lines to them.

contribution to the orbital energy is larger than the Breit contribution. Although our QED contributions to orbital energy are similar to those presented in Ref. 59 (see Table VIII), our Breit contributions are much different from those in Ref. 59. Our Breit contributions to valence $n s$ orbital energy are 0.342 $\mathrm{meV}, 0.345 \mathrm{meV}, 0.688 \mathrm{meV}, 5.616 \mathrm{meV}, 13.501 \mathrm{meV}$, and $37.384 \mathrm{meV}$ for ${ }_{37} \mathrm{Rb},{ }_{55} \mathrm{Cs},{ }_{87} \mathrm{Fr},{ }_{47} \mathrm{Ag},{ }_{79} \mathrm{Au}$, and ${ }_{111} \mathrm{Rg}$, respectively.

From the point of view of existing molecular codes, such as Dirac code, ${ }^{60}$ it is interesting to see the Gaunt to leading QED contributions ratio for $s$ subshells-see Fig. 6. For $1 s$ orbitals, $R^{G / Q E D} \simeq 2$ with weak dependence with $Z$. This ratio grows down as $n$ increases and depends on $Z$ for $n>2$. For $2 s$ orbitals, $R^{G / Q E D} \simeq 1.5$.

QED contributions to orbital energy $(\mathrm{eV})$ of the valence $n s$-subshell for group 1 and 11 atoms are presented in Table VIII and compared to other theoretical data available in the literature. Though the approach to calculate the VP correction is well established, various methods to calculate SE correction are used. In the studies of Dzuba et al. ${ }^{61,62}$ the quantum defect method is used; in the study of Pyykkö et $a l, .{ }^{59}$ the SE/VP ratio method is used; in the work of
Labzowsky et al. ${ }^{63}$ the multiple-commutator method (MCM) is used; in the work of Pyykkö and Zhao ${ }^{64}$ the local Gaussiantype operator (LGO) is used; in the work of Eliav et al. ${ }^{65}$ the Welton picture is applied; in the studies of Dinh et al. ${ }^{66}$ Goidenko, ${ }^{67}$ Thierfelder and Schwerdtfeger, ${ }^{44}$ and Schwerdtfeger et al. ${ }^{68}$ the various approaches to the effective nonlocal SE operator (ENLO) $)^{43}$ are used; in the studies of Tupitsyn et al. ${ }^{69}$ and Pašteka et al. ${ }^{70}$ the various approaches to model Lamb shift operator (MLSO) ${ }^{45}$ are carried out. Our results are performed following three approaches, which differ only in the evaluation of SE screening. In "model 1," the SE is calculated according to Eq. (10). In "model 2," the SE is calculated according to Eq. (12). In "model 3," the QED contribution is calculated by means of the MLSO model (using QEDMOD code ${ }^{45}$ ). In order to keep consistency, calculations performed in each model are based on wavefunctions generated by Grasp2K code. "DF" and "MP" refer to different electronic wavefunctions used in the work of Pyykkö et $a l .{ }^{59}$; they are DF-Dirac-Fock wavefunctions generated by MCDFGME code, and MP-valence wavefunctions obtained using the local effective potential of Salvat et al. "71 "(1)," "(2)," and "(3)" refer to different models used in the work of 


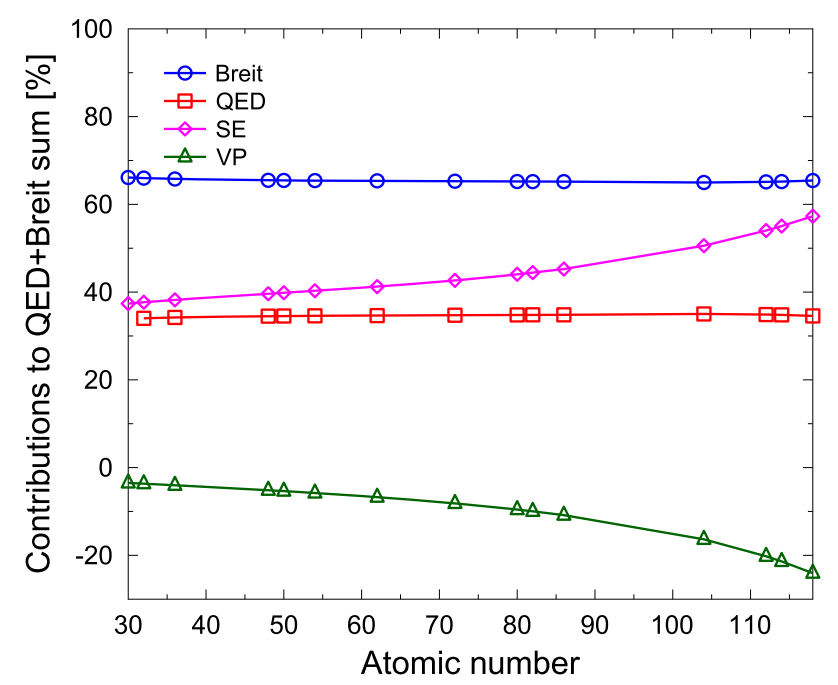

$(1 \mathrm{~s})$

(a)

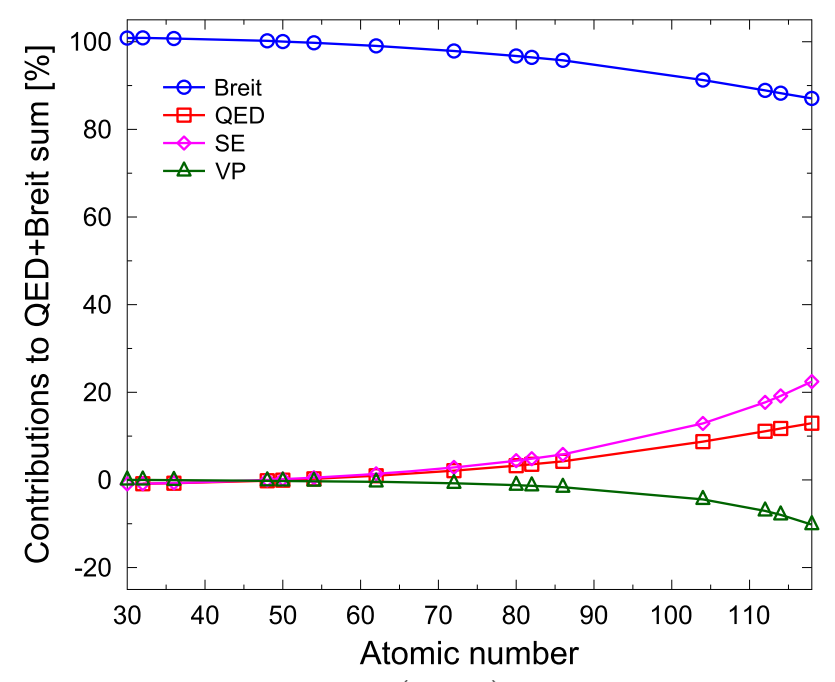

$\left(2 \mathrm{p}_{1 / 2}\right)$

(c)

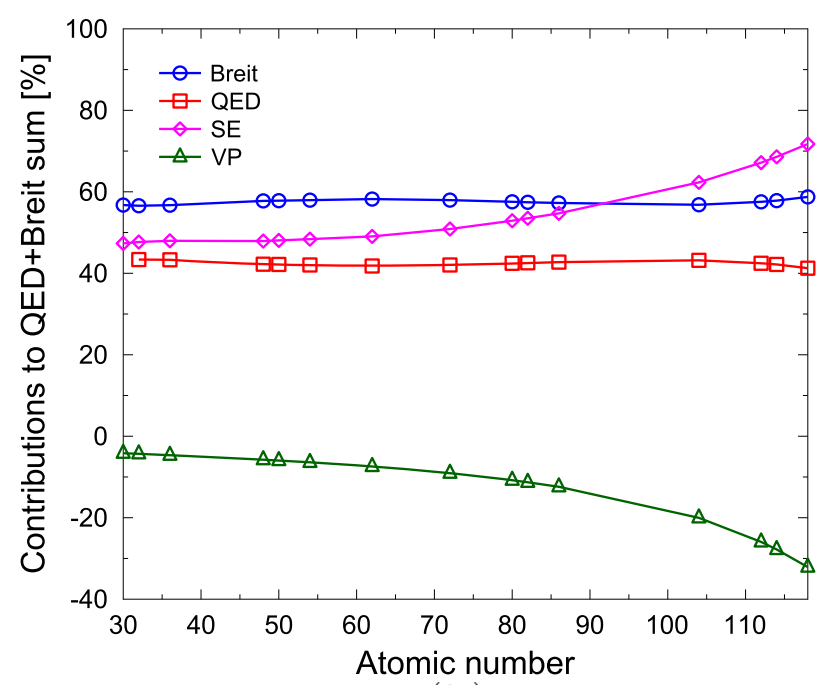

(2s)

(b)

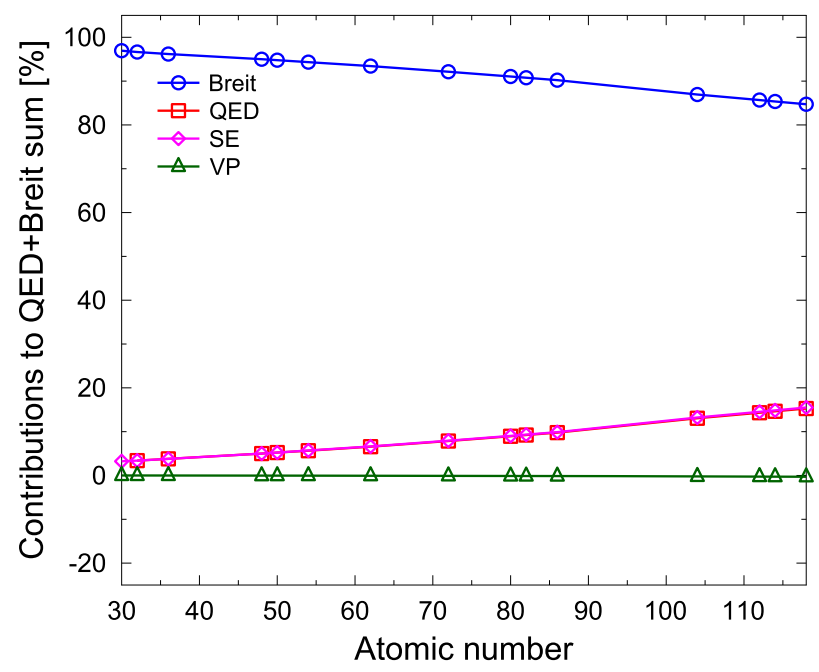

$\left(2 \mathrm{p}_{3 / 2}\right)$

(d)

FIG. 4. Sum of leading QED plus Breit contributions to the Dirac-Coulomb orbital energy for selected subshells.

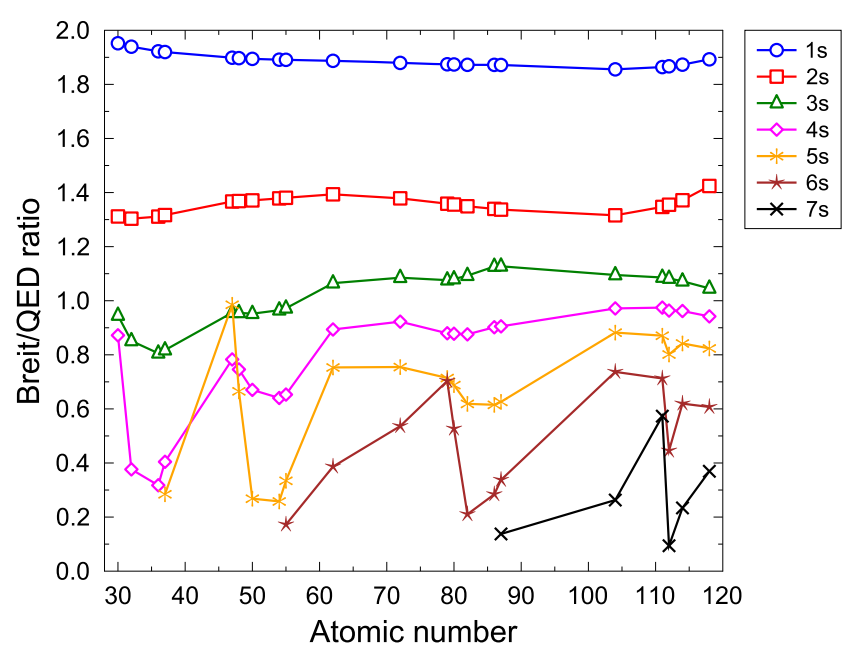

FIG. 5. Asymptotic behavior of Breit to leading QED contributions ratio for $s$ subshells.
Labzowsky et al. ${ }^{63}$; the following electronic wavefunctions are used: (1)—Dirac-Fock, (2)—Dirac-Slater with $\alpha_{x}$ fitted to $\varepsilon_{n s}=\varepsilon_{n s}^{D F}$, and (3)-Dirac-Slater with $\alpha_{x}$ fitted to $\varepsilon_{n s}=-I$ ( $\alpha_{x}$ is the Slater exchange parameter and $I$ is the experimental first ionization potential). "PT" and "DHF" refer to different models used in the work of Goidenko ${ }^{67}$; "PT" means that VP and SE values are calculated perturbatively, "DHF" means that SE and VP energy corrections are included inside the DHF procedure. "DF-DC," "DF-DCB," and "CI-MBPT" refer to different models used in the work of Tupitsyn et al. ${ }^{69}$; "DFDC" and "DF-DCB" mean that the model QED operator was included in the Dirac-Coulomb (DC) and Dirac-CoulombBreit (DCB) Hamiltonians; "CI-MBPT" means additional CIMBPT electron-electron correlation calculations. "D2 "DC-HF" and "CCSD" refer to different models used in the work of Pašteka et al. ${ }^{70}$; "DC-HF" means the Dirac-Fock wavefunction used and "CCSD" means the Dirac-Fock wavefunction 
TABLE VIII. QED contributions to orbital energy or QED correction to ionization potential (meV) of the valence $n s$-subshells for group 1 and 11 atoms.

\begin{tabular}{|c|c|c|c|c|c|c|c|c|}
\hline & $\begin{array}{c}\text { SE } \\
\text { model }\end{array}$ & Wavefunction $^{\mathrm{a}}$ & $\begin{array}{c}{ }_{37} \mathrm{Rb} \\
5 \mathrm{~s}\end{array}$ & $\begin{array}{c}{ }_{55} \mathrm{Cs} \\
6 \mathrm{~s}\end{array}$ & $\begin{array}{c}{ }_{87} \mathrm{Fr} \\
7 \mathrm{~s}\end{array}$ & $\begin{array}{c}{ }_{47} \mathrm{Ag} \\
5 \mathrm{~s}\end{array}$ & $\begin{array}{c}{ }_{79} \mathrm{Au} \\
6 \mathrm{~s}\end{array}$ & $\begin{array}{c}111 \mathrm{Rg} \\
7 \mathrm{~s}\end{array}$ \\
\hline \multicolumn{9}{|l|}{ Present: } \\
\hline Model 1 & Welt. & DF & 1.276 & 2.158 & 5.318 & 6.010 & 20.899 & 65.226 \\
\hline Model 2 & Dens. & DF & 1.237 & 2.099 & 5.419 & 5.831 & 20.896 & 72.658 \\
\hline Model 3 & MLSO & DF & 1.197 & 1.959 & 4.961 & 5.567 & 19.096 & 57.788 \\
\hline \multicolumn{9}{|l|}{ Other: } \\
\hline Dzuba et al..$^{61}$ & q.def. & RHF & & & 9.5 & & & \\
\hline Dzuba et al. ${ }^{62}$ & q.def. & RHF & & 3.5 & & & & \\
\hline Pyykkö et al. ${ }^{59}$ (DF) & Ratio & DF & 1.027 & 1.923 & 4.754 & 5.506 & 18.42 & 56.56 \\
\hline Pyykkö et al. ${ }^{59}$ (MP) & Ratio & MP & 2.092 & 3.398 & 7.507 & 8.132 & 26.01 & \\
\hline Labzowsky et al. ${ }^{63}$ (1) & $\mathrm{MCM}$ & DF & 1.1 & 1.85 & 4.6 & 5.4 & 17.5 & 54.7 \\
\hline Labzowsky et al. ${ }^{63}$ (2) & $\mathrm{MCM}$ & DHS & 1.49 & 2.35 & 5.44 & 6.98 & 21.2 & 61 \\
\hline Labzowsky et al. ${ }^{63}$ (3) & $\mathrm{MCM}$ & DHS & 1.99 & 3.3 & 7.58 & 9.32 & 25.5 & \\
\hline Pyykkö and Zhao ${ }^{64 ~ b}$ & LGO & DF & 1.307 & 2.052 & 5.029 & 5.907 & 19.301 & 65.566 \\
\hline Eliav et al. ${ }^{65}$ & Welt. & DF & 1.28 & 2.23 & 3.57 & & & \\
\hline Dinh et al. ${ }^{66}$ & ENLO & RHF & & 2.0 & 4.5 & & & \\
\hline Goidenko $^{67}(\mathrm{PT})$ & ENLO & DF & & & & 5.5 & & 56.3 \\
\hline Goidenko $^{67}$ (DHF) & ENLO & DF & & & & 6.52 & & 62.6 \\
\hline Thierfelder and Schwerdtfeger ${ }^{44}$ & ENLO & DF & 1.298 & 2.049 & 4.675 & 6.484 & 21.15 & 52.91 \\
\hline Schwerdtfeger et al. ${ }^{68}$ & ENLO & DF & & & & & 21.6 & \\
\hline Tupitsyn et al. ${ }^{69}$ (DF-DC) & MLSO & DF & 1.269 & 2.02 & 4.76 & & & \\
\hline Tupitsyn et al. ${ }^{69}$ (DF-DCB) & MLSO & DF & 1.264 & 2.006 & 4.689 & & & \\
\hline Tupitsyn et al. ${ }^{69}$ (CI-MBPT) & MLSO & DF & 1.428 & 2.254 & 5.073 & & & \\
\hline Pašteka et al..$^{70}(\mathrm{DC}-\mathrm{HF})$ & MLSO & DF & & & & & 21.2 & \\
\hline Pašteka et al. ${ }^{70}$ (CCSD) & MLSO & DF & & & & & 25.8 & \\
\hline
\end{tabular}

${ }^{\mathrm{a}} \mathrm{DF}$, DHS, RHF, and MP means the Dirac-Hartree-Fock, Dirac-Hartree-Fock-Slater, Relativistic Hartree-Fock, and model-potential wavefunction, respectively.

${ }^{\mathrm{b}} \mathrm{SE}$ values from the work of Pyykkö and Zhao ${ }^{64}$ and VP values from the work of Pyykkö et al. ${ }^{59}$

used plus additional contributions from the coupled cluster (CC) method (single and double excitations).

Ionization potential (IP) of coinage or alkali metals can be a benchmark for testing QED estimation for valence shells. ${ }^{7}$ QED contributions to orbital energy of the valence $n s$-subshell is quiet good estimation of QED correction to IP (having opposite sign) because of small relaxation of the singleoccupied $n s$ valence orbital. IP of coinage metals is strongly

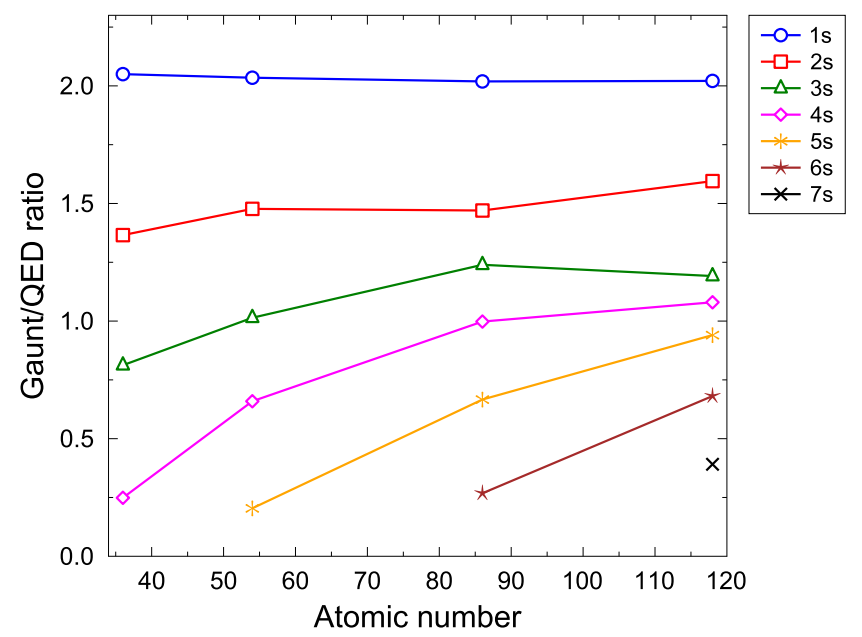

FIG. 6. Asymptotic behavior of Gaunt to leading QED contributions ratio for $s$ subshells. affected by electron correlation effects, ${ }^{73}$ which overwhelms QED contribution. However, the recent development of highresolution spectroscopy, on the one hand, and extensive electron correlation calculations, on the other hand, give a chance for testing expected QED contribution for valence shells. Earlier theoretical studies focusing on IP of $\mathrm{Au}$ underestimated experimental $\mathrm{IP}^{74}$ by $140 \mathrm{meV},{ }^{75}$ by $103 \mathrm{meV},{ }^{73}$ by 19 or 41 $\mathrm{meV},{ }^{76}$ and by $15 \mathrm{meV} .{ }^{77}$ They used various implementation of CC methods in order to proper counting correlation effects. These differences have a comparable order to expected QED contribution to IP but opposite signs. Recently, Pašteka et al. ${ }^{70}$ provided the CC-based result that, excluding QED contribution, overestimates experimental IP by $29.0 \mathrm{meV}$. This value has comparable size to our values for Au in Table VIII, and also to $-25.8 \mathrm{meV}$ QED correction to IP provided by Pašteka et al. ${ }^{70}$ Similar studies were done for IP of Rb, Cs, Fr, and Ag. In the case of $\mathrm{Rb}$, the difference between theoretical and experimental values of IP (without including QED corrections) is $4.8 \mathrm{meV}^{65}$ In the case of $\mathrm{Cs}$, this difference is $9.7,{ }^{78} 1.7,{ }^{65}$ and $7.7 \mathrm{meV}^{77}$ In the case of Fr, this difference is $10.1,{ }^{78}$ $-1.0,{ }^{65}$ and $6.3 \mathrm{meV}^{77}$ In the case of $\mathrm{Ag}$, this difference is $-35.1^{79}$ and $-64.4 \mathrm{meV}^{77}$ The theoretical-experimental difference from the work of Eliav et al. ${ }^{65}$ in the case of Cs, and from the work of Pathak et al. ${ }^{77}$ in the case of $\mathrm{Fr}$, is close to our estimation for QED correction. In the cases of $\mathrm{Rb}$ and $\mathrm{Ag}$, there is no simple conclusion because the theoretical-experimental difference is larger or of opposite sign that expected QED 
contributions. In these cases, more extensive electron correlation calculations are required. Since the QED correction to IP is expected to be a missing part responsible for reducing theoretical-experimental difference, our QED estimation may be a test for examining the quality of extensive electron correlation calculations.

\section{CONCLUSIONS}

In this study, we investigated QED effects on individual atomic orbital energies. Based on the findings presented above, a few main conclusions can be drawn.

(1) The Z-dependence of QED contributions to orbital energies has been evaluated by fitting QED contributions to the orbital $n s$ and $n p$ energies by the $a \times Z^{b}$ power function. The $a$ and $b$ coefficients may be used to estimate the QED effect on inner molecular orbitals. We observe some clean patterns: (i) for given $n, \varepsilon_{n s}^{Q E D} \gg \varepsilon_{n p_{1 / 2}}^{Q E D}$ $\approx \varepsilon_{n p_{3 / 2}}^{Q E D}$; (ii) $\varepsilon_{n p_{1 / 2}}^{Q E D}<\varepsilon_{n p_{3 / 2}}^{Q E D}$ for $Z \leq 86$ but $\varepsilon_{n p_{1 / 2}}^{Q E D}>\varepsilon_{n p_{3 / 2}}^{Q E D}$ for higher $Z$.

(2) The Breit plus QED contributions to orbital energies and the Breit to QED ratio per orbital have been studied. For $n s$ subshells, the Breit and QED contributions are of comparative size, but for $n p$ and $n d$ subshells the Breit contribution takes major part of the QED+Breit sum. We observe that for given $n, \varepsilon_{n s}^{Q E D+B} \approx \varepsilon_{n p_{1 / 2}}^{Q E D+B}$ $>\varepsilon_{n p_{3 / 2}}^{Q E D+B}$. The Lamb shift is attributed to the nucleuselectron system, and the Breit interaction is attributed to the electron-electron system. However, the $R^{B / Q E D}$ ratio for inner $n s$ subshells seems to be almost independent from $Z$. One can suggest that the QED and the Breit contributions to the orbital energy should be considered together within the Lamb shift term.

(3) There is a behavior of Breit to leading QED contributions ratio $\left(R^{B / Q E D}\right)$ with $Z$ increasing for $n s$ subshells. For inner shells, the $R^{B / Q E D}$ ratio is almost independent of $Z$ : for $1 s$ subshells, it is about 1.9-2.0; for $2 s$ subshells, it is about 1.3-1.5; and for $3 s$ subshells, it is about 1.0-1.1.

(4) We presented our calculations for QED contributions to the orbital energy of the valence $n s$-subshell for group 1 and 11 atoms and compared them with other numbers in the literature. We discussed about the reliability of these numbers by using experimental IP data. Our numbers fit well to the differences between theoretical and experimental data, which are expected to be related to the QED effect.

\section{SUPPLEMENTARY MATERIAL}

See supplementary material for a set of ratios between energy contribution of self-energy, vacuum-polarization, Breit, and Dirac-Coulomb terms: the SE to VP energy contributions ratio $\varepsilon_{n l}^{\mathrm{SE}} / \varepsilon_{n l}^{V P}$, the $\mathrm{SE}$ to Breit energy contributions ratio $\varepsilon_{n l}^{\mathrm{SE}} / \varepsilon_{n l}^{B}$, the $\mathrm{VP}$ to Breit energy contributions ratio $\varepsilon_{n l}^{V P} / \varepsilon_{n l}^{B}$, the leading QED to Breit energy contributions ratio $\varepsilon_{n l}^{Q E D} / \varepsilon_{n l}^{B}$, and the leading QED energy contributions to Dirac-Coulomb orbital energy ratio $\varepsilon_{n l}^{Q E D} / \varepsilon_{n l}^{D C}$.

\section{ACKNOWLEDGMENTS}

We gratefully acknowledge partial support from SGCyTUNNE and the Argentinian National Research Council for Science and Technology (CONICET, Grant No. PIP 11220090100654).

${ }^{1}$ P. J. Mohr, G. Plunien, and G. Soff, Phys. Rep. 293, 227 (1998).

${ }^{2}$ S. G. Karshenboim, Phys. Rep. 422, 1 (2005).

${ }^{3}$ A. Rudziński, M. Puchalski, and K. Pachucki, J. Chem. Phys. 130, 244102 (2009).

${ }^{4}$ V. A. Yerokhin, K. Pachucki, Z. Harman, and C. H. Keitel, Phys. Rev. Lett. 107, 043004 (2011).

${ }^{5}$ V. A. Yerokhin, K. Pachucki, Z. Harman, and C. H. Keitel, Phys. Rev. A 85, 022512 (2012).

${ }^{6}$ C. A. Giménez, K. Kozioł, and G. A. Aucar, Phys. Rev. A 93, 032504 (2016).

${ }^{7}$ P. Pyykkö, Chem. Rev. 112, 371 (2012).

${ }^{8}$ G. A. Aucar, Phys. Chem. Chem. Phys. 16, 4420 (2014).

${ }^{9}$ W. Liu, Phys. Rep. 537, 59 (2014).

${ }^{10}$ A. F. Maldonado, C. A. Giménez, and G. A. Aucar, J. Chem. Phys. 136, 224110 (2012).

${ }^{11}$ W. E. Lamb, Science 123, 439 (1956).

${ }^{12}$ A. N. Artemyev, V. M. Shabaev, V. A. Yerokhin, G. Plunien, and G. Soff, Phys. Rev. A 71, 062104 (2005).

${ }^{13}$ M. Puchalski and K. Pachucki, Phys. Rev. A 92, 012513 (2015).

${ }^{14}$ M. Puchalski, J. Komasa, and K. Pachucki, Phys. Rev. A: At., Mol., Opt. Phys. 87, 030502(R) (2013).

${ }^{15}$ M. H. Chen, K. T. Cheng, P. Beiersdorfer, and J. Sapirstein, Phys. Rev. A 68, 022507 (2003).

${ }^{16}$ J. D. Gillaspy, J. Instrum. 5, C10005 (2010).

${ }^{17}$ K. T. Cheng, M. H. Chen, W. R. Johnson, and J. Sapirstein, Can. J. Phys. 86, 33 (2008).

${ }^{18}$ J. Biesheuvel, J.-P. Karr, L. Hilico, K. S. E. Eikema, W. Ubachs, and J. C. J. Koelemeij, Nat. Commun. 7, 10385 (2016).

${ }^{19}$ V. I. Korobov, L. Hilico, and J.-P. Karr, Phys. Rev. A 89, 032511 (2014).

${ }^{20}$ G. D. Dickenson, M. L. Niu, E. J. Salumbides, J. Komasa, K. S. E. Eikema, K. Pachucki, and W. Ubachs, Phys. Rev. Lett. 110, 193601 (2013).

${ }^{21}$ P. Pyykkö, K. G. Dyall, A. G. Császár, G. Tarczay, O. L. Polyansky, and J. Tennyson, Phys. Rev. A 63, 024502 (2001).

${ }^{22}$ I. P. Grant, Methods in Computational Chemistry (Springer US, Boston, MA, 1988), Vol. 2, pp. 1-71.

${ }^{23}$ I. P. Grant, Relativistic Quantum Theory of Atoms and Molecules, Springer Series on Atomic, Optical, and Plasma Physics (Springer, New York, NY, 2007), Vol. 40.

${ }^{24}$ I. P. Grant, J. Phys. B: At., Mol. Opt. Phys. 43, 074033 (2010).

${ }^{25}$ I. P. Grant, Adv. Phys. 19, 747 (1970).

${ }^{26}$ O. Gorceix, P. Indelicato, and J.-P. Desclaux, J. Phys. B: At. Mol. Phys. 20, 639 (1987).

${ }^{27}$ P. Indelicato, O. Gorceix, and J.-P. Desclaux, J. Phys. B: At. Mol. Phys. 20, 651 (1987).

${ }^{28}$ G. Breit, Phys. Rev. 34, 553 (1929).

${ }^{29}$ G. Breit, Phys. Rev. 36, 383 (1930).

${ }^{30}$ G. Breit, Phys. Rev. 39, 616 (1932).

${ }^{31}$ I. Lindgren, Relativistic Many-Body Theory: A New Field-Theoretical Approach, Springer Series on Atomic, Optical, and Plasma Physics (Springer-Verlag, New York, 2011), Vol. 63.

${ }^{32}$ L. W. Fullerton and G. A. Rinker, Phys. Rev. A 13, 1283 (1976).

${ }^{33}$ G. Källén and A. Sabry, Det Kongelige Danske Videnskabernes Selskab Matematisk-fysiske meddelelser 29, 1-20 (1955), see http://gymarkiv. sdu.dk/MFM/kdvs/mfm\%2020-29/MFM\%2029-17.pdf.

${ }^{34}$ E. H. Wichmann and N. M. Kroll, Phys. Rev. 101, 843 (1956).

${ }^{35}$ J. Blomqvist, Nucl. Phys. B 48, 95 (1972).

${ }^{36}$ P. J. Mohr, Phys. Rev. Lett. 34, 1050 (1975).

${ }^{37}$ P. J. Mohr, Phys. Rev. A 26, 2338 (1982).

${ }^{38}$ P. J. Mohr and Y.-K. Kim, Phys. Rev. A 45, 2727 (1992).

${ }^{39}$ T. A. Welton, Phys. Rev. 74, 1157 (1948).

${ }^{40}$ P. Indelicato and J.-P. Desclaux, Phys. Rev. A 42, 5139 (1990).

${ }^{41}$ P. Indelicato, J. P. Santos, S. Boucard, and J.-P. Desclaux, Eur. Phys. J. D 45, 155 (2007).

${ }^{42}$ J. A. Lowe, C. T. Chantler, and I. P. Grant, Radiat. Phys. Chem. 85, 118 (2013).

${ }^{43}$ V. V. Flambaum and J. S. M. Ginges, Phys. Rev. A 72, 052115 (2005).

${ }^{44}$ C. Thierfelder and P. Schwerdtfeger, Phys. Rev. A 82, 062503 (2010). 
${ }^{45}$ V. M. Shabaev, I. I. Tupitsyn, and V. A. Yerokhin, Comput. Phys. Commun. 189, 175 (2015).

${ }^{46}$ V. M. Shabaev, I. I. Tupitsyn, and V. A. Yerokhin, Comput. Phys. Commun. 223, 69 (2018).

${ }^{47}$ K. G. Dyall, J. Chem. Phys. 139, 021103 (2013).

${ }^{48}$ P. Indelicato and E. Lindroth, Phys. Rev. A 46, 2426 (1992).

${ }^{49} \mathrm{P}$. Indelicato and J.-P. Desclaux, MCDFGME, a multiconfiguration Dirac-Fock and General Matrix Elements program, release, 2005, http://dirac.spectro.jussieu.fr/mcdf.

${ }^{50}$ J.-P. Desclaux, Comput. Phys. Commun. 9, 31 (1975).

${ }^{51}$ J.-P. Desclaux, Comput. Phys. Commun. 35, C288 (1984).

${ }^{52}$ K. Kozioł, C. A. Giménez, and G. A. Aucar, J. Chem. Phys. 148, 044113 (2018).

${ }^{53}$ W. R. Johnson and G. Soff, At. Data Nucl. Data Tables 33, 405 (1985).

${ }^{54}$ V. A. Yerokhin and V. M. Shabaev, J. Phys. Chem. Ref. Data 44, 033103 (2015).

${ }^{55}$ H. A. Bethe, Phys. Rev. 72, 339 (1947).

${ }^{56} \mathrm{See}$ http://scidavis.sourceforge.net for SciDAVis.

${ }^{57}$ N. Gaston, P. Schwerdtfeger, and W. Nazarewicz, Phys. Rev. A 66, 062505 (2002).

${ }^{58}$ K. Pachucki, Phys. Rev. A: At., Mol., Opt. Phys. 53, 2092 (1996).

${ }^{59}$ P. Pyykkö, M. Tokman, and L. N. Labzowsky, Phys. Rev. A 57, R689 (1998).

${ }^{60}$ DIRAC, a relativistic ab initio electronic structure program, Release DIRAC17, 2017, written by L. Visscher, H. J. Aa. Jensen, R. Bast, and T. Saue, with contributions from V. Bakken, K. G. Dyall, S. Dubillard, U. Ekström, E. Eliav, T. Enevoldsen, E. Faßhauer, T. Fleig, O. Fossgaard, A. S. P. Gomes, E. D. Hedegård, T. Helgaker, J. Henriksson, M. Iliaš, Ch. R. Jacob, S. Knecht, S. Komorovský, O. Kullie, J. K. Lærdahl, C. V. Larsen, Y. S. Lee, H. S. Nataraj, M. K. Nayak, P. Norman, G. Olejniczak, J. Olsen, J. M. H. Olsen, Y. C. Park, J. K. Pedersen, M. Pernpointner, R. di Remigio, K. Ruud, P. Sałek, B. Schimmelpfennig, A. Shee, J. Sikkema, A. J. Thorvaldsen, J. Thyssen, J. van Stralen, S. Villaume, O. Visser, T. Winther, and S. Yamamoto (see http://www.diracprogram.org).

${ }^{61}$ V. A. Dzuba, V. V. Flambaum, and O. P. Sushkov, Phys. Lett. A 95, 230 (1983).
${ }^{62}$ V. A. Dzuba, V. V. Flambaum, and O. P. Sushkov, J. Phys. B: At. Mol. Phys. 16, 715 (1983).

${ }^{63}$ L. N. Labzowsky, I. A. Goidenko, M. Tokman, and P. Pyykkö, Phys. Rev. A 59, 2707 (1999).

${ }^{64}$ P. Pyykkö and L.-B. Zhao, J. Phys. B: At., Mol. Opt. Phys. 36, 1469 (2003).

${ }^{65}$ E. Eliav, M. J. Vilkas, Y. Ishikawa, and U. Kaldor, Chem. Phys. 311, 163 (2005).

${ }^{66}$ T. H. Dinh, V. A. Dzuba, V. V. Flambaum, and J. S. M. Ginges, Phys. Rev. A 78, 022507 (2008).

${ }^{67}$ I. A. Goidenko, Eur. Phys. J. D 55, 35 (2009).

${ }^{68}$ P. Schwerdtfeger, L. F. Pašteka, A. Punnett, and P. O. Bowman, Nucl. Phys. A 944, 551 (2015).

${ }^{69}$ I. I. Tupitsyn, D. V. Mironova, A. V. Malyshev, and V. M. Shabaev, Nucl. Instrum. Methods Phys. Res., Sect. B 408, 76 (2017).

${ }^{70}$ L. F. Pašteka, E. Eliav, A. Borschevsky, U. Kaldor, and P. Schwerdtfeger, Phys. Rev. Lett. 118, 023002 (2017).

${ }^{71}$ F. Salvat, J. D. Martínez, R. Mayol, and J. Parellada, Phys. Rev. A 36, 467 (1987).

${ }^{72}$ M. G. Kozlov, S. G. Porsev, M. S. Safronova, and I. I. Tupitsyn, Comput. Phys. Commun. 195, 199 (2015).

${ }^{73}$ P. Neogrády, V. Kellö, M. Urban, and A. J. Sadlej, Int. J. Quantum Chem. 63, 557 (1997).

${ }^{74} \mathrm{See}$ https://www.nist.gov/pml/ground-levels-and-ionization-energies-neut ral-atoms for Ground Levels and Ionization Energies for the Neutral Atoms, accessed 01-10-2018.

${ }^{75}$ E. Eliav, U. Kaldor, and Y. Ishikawa, Phys. Rev. A 49, 1724 (1994).

${ }^{76}$ A. Landau, E. Eliav, Y. Ishikawa, and U. Kaldor, J. Chem. Phys. 121, 6634 (2004).

${ }^{77}$ H. Pathak, S. Sasmal, M. K. Nayak, N. Vaval, and S. Pal, J. Chem. Phys. 145, 074110 (2016)

${ }^{78}$ V. A. Dzuba, V. V. Flambaum, and J. S. M. Ginges, Phys. Rev. A 63, 062101 (2001).

${ }^{79}$ M. K. Nayak, R. K. Chaudhuri, S. Chattopadhyay, and U. S. Mahapatra, J. Mol. Struct.: THEOCHEM 768, 133 (2006). 